\title{
O ACOMPANHANTE DE CRIANÇA SUBMETIDA À CIRÚRGIA CARDÍACA: CONTRIBUIÇÃO PARA A ENFERMAGEM ${ }^{a}$
}

\author{
The Companion of the Child that Suffered a \\ Cardiac Surgery: Contribution for the Nursing \\ El Acompañante del Niño Sometido a la \\ Cirugía Cardiaca: Contribución para la Enfermería
}

Dominique Cavalcanti Méllo

Benedita Maria Rêgo Deusdará Rodrigues²

\begin{abstract}
Resumo
A experiência profissional no cuidar de crianças com cardiopatias congênitas nos levou a realizar o estudo cujo objeto foi o significado do atendimento no ambulatório de cirurgia cardíaca pediátrica, e o objetivo, apreender o típico da ação do acompanhante que traz a criança ao atendimento no ambulatório de cirurgia cardíaca. Para tanto, se utilizou a metodologia qualitativa do tipo exploratório-descritivo baseada na fenomenologia sociológica de Alfred Schutz cujos sujeitos foram os acompanhantes de crianças que realizaram cirurgia cardíaca e que retornaram para a revisão ambulatorial. As falas foram captadas com a entrevista fenomenológica, utilizando-se as seguintes questões: Qual o seu parentesco com a criança? 0 que espera da consulta no ambulatório? 0 típico da ação da acompanhante de crianças que se submeteu à cirurgia cardíaca é "obter uma boa notícia". 0 estudo nos fez refletir sobre a importância da inclusão do acompanhante como cliente do fazer cotidiano da Enfermagem Pediátrica.
\end{abstract}

Palavras-chave: Enfermagem Pediátrica. Cardiopatias Congênitas. Acompanhantes de Pacientes. Pesquisa Qualitativa.

\begin{abstract}
The professional experience tacking care of children with congenital cardiopathies took us to realize a study whose object was the significance of the attending in the cardiac pediatric surgery ambulatory, and the objective of apprehending the typical action of the companion that bring the child to the attending in the cardiac pediatric surgery ambulatory. Therefore, was used the qualitative methodology of the type exploratorydescription based on the sociological phenomenology of Alfred Schutz whose subjects were the companions of children that realized a cardiac surgery and came back to the ambulatory review. The words were sucked in with the phenomenological interview, using these questions: Which are your kinship with the child? e, what do you expect from the ambulatory meeting? The typical action of the companion of children that were submitted to cardiac surgery is "obtain a good news". The study made us to reflect about the importance of including the companion as a client of a routine make of a pediatric nursing.
\end{abstract}

\section{Resumen}

La experiencia profesional que cuida de niños con cardiopatías congénitas nos llevó a realizar el estudio cuyo objeto fue el significado de la atención de ambulatoria de cirugía cardiaca pediátrica, con el objetivo de aprehender la tipicidad de la acción del acompañante que trae el niño al ambulatorio de cirugía cardiaca. Para esto, se utilizó la metodología cualitativa del tipo exploratorio-descriptivo basada en la fenomenología sociológica de Alfred Schutz cuyos sujetos fueran los acompañantes de niños que realizaran cirugía cardiaca y que volvieran para la revisión ambulatorial. Las conversaciones fueron obtenidas por medio de entrevista fenomenológica, utilizándose de las siguientes preguntas: ¿Cual es su parentesco con el niño? y, ¿Que deseas de la consulta de ambulatorio? Es típico de la acción del acompañante del niño que se sometió a la cirugía cardiaca "obtener una buena noticia". El estudio nos hizo refletir sobre la relevancia de la inclusión del acompañante como cliente en la rutina diaria de la enfermería pediátrica.
Keywords: Pediatric Nursing. Heart Defects, Congenital . Patient Escort Service. Qualitative Research.
Palabras clave: Enfermería pediátrica. Cardiopatías Congénitas. Acompañantes de Pacientes. Investigación Cualitativa. 


\section{INTRODUÇÃO}

Durante experiência profissional, em uma instituição hospitalar especializada em cardiologia, cuidando de crianças de todas as idades com cardiopatias congênitas, surgiram alguns questionamentos quanto a que tipo de informação os acompanhantes (segundo o Ministério da Saúde, são os representantes da rede social do paciente que o acompanham durante toda a permanência no ambiente hospitalar) ${ }^{1}$ recebem quando chegam, ao saber que a criança será operada e o que pode vir a acontecer com elas.

Esse convívio mostrou a importância do momento da internação para a aproximação do Enfermeiro com o acompanhante porque possibilita o processo de recepção e orientação quanto à rotina hospitalar e aos procedimentos realizados com a criança. Entendemos que a interação do profissional com o acompanhante permite reduzir o estresse tão comum no momento da internação, propiciando uma maior segurança no acompanhamento da criança durante esse período.

A oportunidade de recepcionar essas crianças para 0 ato cirúrgico nos fez perceber a angústia desses acompanhantes ao deixarem suas crianças entrarem por uma porta, a do centro cirúrgico, pois eles não sabem o que pode acontecer lá dentro. Esses acompanhantes por vezes permanecem no corredor na tentativa de saber o que está acontecendo com suas crianças.

Nesse sentido, Sales ${ }^{2}$ refere, em seu estudo sobre o sentimento das mães de crianças submetidas à primeira cirurgia cardíaca, que dos resultados encontrados o medo foi a categoria que caracteriza o sentimento real dessas mães e que 0 "diagnóstico de enfermagem foi definido como: fuga diante de uma condição de ameaça [...]".

Assim sendo, vale lembrar que esta instituição é especializada em Cardiologia, possuindo uma unidade pediátrica para 0 atendimento de crianças a partir da fase neonatal.

Acreditamos que a forma de atendimento dessas pessoas, acompanhante e criança, deveria ser diferenciada, pois algumas passarão o resto da vida com o acompanhamento clínico, apesar da correção cirúrgica. Essas pessoas necessitam de um guia, alguém que diga como proceder, o que fazer, para que se sintam cuidadas, seguras e que a internação da criança seja o mais proveitosa possível.

Gomes e Lunardi ${ }^{3}$ nos chama a atenção para um modo de cuidar que envolva o acompanhante, onde a equipe de enfermagem atua e planeja a assistência à criança juntamente com o mesmo, para que tenha condições de assumir funções ativas no cuidado à criança.

Nesse sentido, o Estatuto da Criança e do Adolescente ${ }^{4}$, no seu Art. 12, destaca que "os estabelecimentos de atendimento à saúde deverão proporcionar condições para a permanência em tempo integral de um dos pais ou responsável, nos casos de internação de criança ou adolescente". Contudo, as instituições não estão preparadas para receber os acompanhantes destas crianças $5: 260$.

Apesar de tudo isso, os acompanhantes têm que enfrentar esta situação, e o sentimento mais comum é o medo do desconhecido. 0 que estes acompanhantes esperam da equipe de profissionais? Será que o que dizemos a eles é esclarecedor? Alguns destes acompanhantes começam a fazer uma série de questionamentos, o que algumas vezes traz desconforto, e eles acabam sendo considerados inconvenientes pelos profissionais? ${ }^{2}$.

Segundo Gomes e Lunardi3:29, "a família é um cliente que possui necessidades e que procura na enfermagem apoio e ajuda na busca por sua saúde e sua autonomia". Nesse sentido, Pettengill ${ }^{6}$ corrobora Gomes e Lunardi ${ }^{3}$ quando, em estudo realizado para conceituar a vulnerabilidade da família que experiencia a internação de um filho doente, apresenta uma categoria que emergiu das entrevistas "sentindo-se ameaçada em sua autonomia".

No cotidiano profissional percebemos que a família não é o foco da atenção, e sim a criança. Se o acompanhante questiona muito e demanda tempo e atenção, é considerado inconveniente. Os profissionais se esquecem que esta criança vai para casa e que vai precisar dos cuidados deste familiar, que, se sair do hospital sem uma orientação correta e esclarecida, não vai aderir ao tratamento e certamente vai retornar e reinternar por falta de medicamento ou com uma ferida pós-cirúrgica com infecção, por exemplo.

Acreditamos que os profissionais de saúde do pós-operatório de cirurgia cardíaca precisarão assumir a responsabilidade de orientar estes acompanhantes quanto à situação pré e pósoperatória destas crianças. É sabido que essas crianças retornam ao ambulatório da instituição para acompanhamento cardiológico, mas quem assume as questões que os acompanhantes demandam?

Desta forma emergiu, ainda, o seguinte questionamento:

Como é realizado o atendimento das crianças no ambulatório de cirurgia cardíaca?

As crianças acometidas de cardiopatias congênitas na maioria das vezes são consideradas portadoras de patologia crônica, reforçando a necessidade de orientação dos acompanhantes para dar seqüência no tratamento.

Bowlby em "A Teoria do Apego" enfatiza a função biológica dos laços emocionais íntimos, especialmente entre a mãe e 0 bebê, e a influência dos cuidadores principais para 0 desenvolvimento da criança ${ }^{6}$. Estudos sobre apego e enfermidade em crianças portadoras de doença crônica têm mostrado resultados inconsistentes na relação de apego que se estabelece entre mãe e filho. Outros autores encontraram menos apego seguro em crianças com doença cardíaca congênita e crianças com fibrose cística aos 12 e 18 meses de idade, quando comparadas às crianças saudáveis 8 .

Entretanto Souza, Cosmo e Pimentel ${ }^{9}$, em seu estudo sobre a "terapêutica eficaz para mães de crianças com cardiopatias congênitas", apontaram para a necessidade de realizar um atendimento diferenciado por apresentarem reações psicológicas impactantes e perturbadoras diante da descoberta da doença cardíaca de seus filhos.

Vale ressaltar que o estudo irá contribuir para a assistência de enfermagem direcionada a essa clientela, assim como para a qualidade das informações dadas aos acompanhantes de crianças portadoras de cardiopatias congênitas e que realizaram uma correção cirúrgica. 
A partir do exposto, temos como objeto de estudo o significado do atendimento no ambulatório de cirurgia cardíaca pediátrica, e o objetivo, apreender o típico da ação do acompanhante que traz a criança ao atendimento no ambulatório de cirurgia cardíaca. Como é o seu mundo e como ele vivencia a experiência de ter um familiar que se submeteu a uma cirurgia cardíaca e que está em casa sob seus cuidados. Os sujeitos do estudo foram os acompanhantes de crianças que realizaram a cirurgia cardíaca e retornaram para acompanhamento ambulatorial.

\section{Algumas considerações sobre a Cardiopatia Congênita e a Enfermagem na perspectiva do acompanhante}

As cardiopatias congênitas são malformações - defeitos anatômicos do coração que vão comprometer a sua função. Segundo Lopes e cols. ${ }^{10}$, aproximadamente 8 em cada 1.000 nascidos vivos são crianças portadoras de cardiopatias congênitas, e, dessas, uma ou duas apresentarão situação de emergência no período neonatal. No Congresso Mundial de Cardiologia Pediátrica de 1980 em Londres referiu-se que a probabilidade em países como o Brasil era de 6 em cada 1.000 nascidos vivos. Na maioria das vezes, as cardiopatias são identificadas logo após o nascimento ou durante a infância, porém há casos em que o diagnóstico pode ser feito intraútero, na adolescência ou na vida adulta. 0 diagnóstico pode ser feito através de manifestações clínicas, estudo radiológico, ecocardiográfico e hemodinâmico.

Cabe destacar que desde a década de 1940 ocorreram vários avanços no diagnóstico, no tratamento clínico e cirúrgico das cardiopatias congênitas, o que contribuiu, especialmente, para a identificação destas patologias ${ }^{11}$. Atualmente, os serviços de hemodinâmica (serviços radiológicos que realizam o cateterismo cardíaco diagnóstico e procedimentos como a angioplastia) investigam e realizam procedimentos corretivos em alguns casos.

As cardiopatias congênitas podem ser de dois tipos: cianóticas e acianóticas, e esta diferenciação nos dará a gravidade do quadro.

As cardiopatias que se manifestam no período neonatal são as mais graves. 0 diagnóstico pré-natal vem crescendo e tem permitido melhorar o prognóstico à medida que prevê se a criança apresentará situação de emergência ao nascer ou necessitará de cirurgia imediata no período neonatal. Por exemplo, é importante determinar se a cardiopatia é cianótica ou acianótica e se o suprimento pulmonar ou sistêmico é dependente de canal arterial ${ }^{12}$.

Nesse contexto, vale lembrar que o Estatuto da Criança e do Adolescente $^{4}(E C A)$ veio garantir a presença de a mãe acompanhar o seu filho doente durante a hospitalização. Até os idos de 1990 somente algumas unidades hospitalares permitiam a permanência de um acompanhante para a criança hospitalizada; com o artigo 12 do ECA 4 isto passou a ser uma obrigatoriedade e as instituições não se prepararam para isto, e ainda hoje, após quase vinte anos de implantação do Estatuto, isto é um problema enfocado inclusive em artigos e pesquisa científicas ${ }^{13}$.

Oliveira e Ângelo 14:203 citam Darbyshire, que "enfatiza a necessidade de os profissionais compreenderem as experiências reais vividas pelo familiar acompanhante quando seus filhos estão hospitalizados". No seu artigo, evidenciou uma categoria central, que é "vivenciando com o filho uma passagem difícil e reveladora", em que as mães aprendem que este momento é importante para o cuidado do seu filho e aprendem também convivendo com a dor e o sofrimento, não se permitindo abater-se com a situação.

Assim sendo, Queiroz e Barroso 15:148 destacam que "o perfil do enfermeiro pediátrico além de assistir, orientar/ensinar crianças e famílias para a saúde, também deve gerar impulsos de motivação e auto-estima para a capacidade de desenvolver a qualidade de vida pessoal e familiar", o que nos faz observar a necessidade de qualificar melhor os profissionais que estão ligados a essa área.

No entanto, Gomes e Lunardi Filho ${ }^{3: 31}$ verificaram que as famílias internadas junto com as crianças identificam suas necessidades, mas isto não é valorizado pela equipe de enfermagem quando da implantação dos seus cuidados. Observa-se, então, que existe uma tentativa do familiar em adequar-se à situação de internação, mas parece que não há um envolvimento da equipe de enfermagem, que define qual é o melhor cuidado sem levar em conta as reais necessidades da criança e da família. Os vários conceitos de família ressaltam a importância da mesma na internação de uma criança. As autoras citam "Elsen, relatando que família é o primeiro sistema de cuidado" e que "Leininger define família como sendo um sistema interpessoal formado por seres humanos que interagem por vários motivos". Mas o que percebemos no cotidiano profissional é que a enfermagem não se preocupa com estas definições no momento em que vai prescrever ou realizar os seus cuidados.

Collière ${ }^{16}$, falando sobre o cuidar, nos lembra que o cuidado é algo realizado entre seres humanos desde o início dos tempos quando ainda não tinha escrita. Esses cuidados se transmitem das mães aos filhos, dos adultos para pessoas idosas, criando desde o início dos tempos redes de apoio mútuo e de solidariedade em torno das passagens da vida.

Silveira ${ }^{17: 899}$,em seu estudo sobre "a experiência de interação da família que vivencia a doença e hospitalização da criança", enfatiza que existe a necessidade de se realizarem mais pesquisas sobre esta interação e que o cuidado centrado na família não é uma regra e isto deve ser inserido na abordagem da Enfermagem Pediátrica.

\section{ABORDAGEM TEÓRICO-METODOLÓGICA}

Trata-se de estudo de caráter exploratório-descritivo, com uma metodologia do tipo qualitativa, baseada fundamentalmente na abordagem fenomenológica, cujos sujeitos foram os acompanhantes de crianças que realizaram cirurgia cardíaca e que retornaram para a revisão ambulatorial em um determinado hospital de cardiologia, público, localizado na Cidade do Rio de Janeiro.

Assim, cabe esclarecer que o método fenomenológico caracteriza-se pela "ênfase ao 'mundo da vida cotidiana' um retorno à totalidade do mundo vivido" 18:40. Para Polit e Hungler 19:40, é "uma maneira de pensar sobre as experiências de vida das pessoas".

0 motivo de opção por este método foi o fato de o objeto de estudo em destaque envolver vivências, pois trata de captar a 
natureza das experiências das pessoas, em particular os acompanhantes das crianças com cardiopatias congênitas, que estiveram internadas e retornam para consulta ambulatorial.

Portanto, esta abordagem metodológica se aplica ao estudo e, como nos diz Coltro ${ }^{18: 38}$, "a pesquisa fenomenológica parte da compreensão do viver e não de definições ou conceitos, e é uma compreensão voltada para os significados do perceber, [...]". Compreender, segundo Bello ${ }^{20: 52}$, é "ter consciência do ato de viver, que é o olhar". Para Polit e Hungler ${ }^{19: 41}$, "a meta da pesquisa fenomenológica é descrever a experiência totalmente vivida [...]".

Josgrilberg 21:44 nos explica que "a fenomenologia visa compreender e não explicar a manifestação das coisas e seus significados, mais do que explicar por razões mecânicas, naturais, físicas ou mesmo por processos sociais."

Para Capalbo $22: 26$, a "tarefa da fenomenologia consistirá em mostrar que o outro, o mundo social, cultural, histórico e natural, ao invés de ser 'fato' já constituído para um sujeito, ao contrário, é constituído pelo sujeito".

Para Schutz, o mundo social "se organiza em um sistema de coordenadas na qual eu sou o centro [...], e eu estou aqui e meu semelhante lá". Portanto, "não podemos nunca ocupar, ao mesmo tempo, o mesmo lugar e a mesma posição"22:32.

Capalbo ${ }^{22}$ destaca que o estudo do mundo social se relaciona com a intersubjetividade, ressaltando que, para Schutz, a intersubjetividade se faz pela fenomenologia voltada para a atitude natural. Assim, conclui-se que cada um se mostra para o outro através de sua linguagem, motivos, sentidos e corpo do outro.

Segundo Wagner ${ }^{23}$, para Schutz, a intersubjetividade é uma "categoria que, em geral, se refere ao que é comum a vários indivíduos". A intersubjetividade se ergue a partir das possibilidades de encontros entre pessoas e a capacidade que essas pessoas têm de apreender o outro em um determinado ambiente. Esse ambiente não é o mundo da vida de cada um, é compartilhado com os outros, ou seja, é um mundo comum a todos. Esse ambiente comum fundamenta-se no fato de que os sujeitos motivam-se mutuamente e, se isso não ocorresse, não haveria o Eu nem o Outro, portanto ambos co-existem ${ }^{23,24}$.

0 mundo da vida é tudo que se relaciona às experiências cotidianas. Tudo o que tiver relação com os interesses, negócios, relacionamentos pessoais e profissionais, tratando com pessoas e idealizando projetos ${ }^{23}$.

Portanto, a "situação biográfica" é a sedimentação das experiências vivenciadas acumuladas e que irá determinar como serão as suas atitudes e ações diante das situações que vierem a ocorrer em sua vida. Segundo Rodrigues ${ }^{25: 20}$, "cada pessoa interpreta a realidade do senso comum, segundo a perspectiva de seus interesses particulares".

Segundo Panizza 26: 130, "a ação é a conduta humana projetada pelo agente de maneira autoconsciente"; é um ato motivado e pode ser dividida em dois tipos que vão variar de acordo com a posição do ator, ou seja, se a resposta da ação é para o futuro ou para o passado ${ }^{23}$.

A orientação da ação futura nos dá uma idéia da intencionalidade da ação. Segundo Schutz ${ }^{22,23,26}$, "o motivopara é, portanto, um contexto de significados que se constrói sobre o contexto de experiências disponíveis no momento da projeção". 0 motivo - para refere-se a algo que se quer realizar, objetivos que se pretende alcançar ${ }^{24}$.

Por outro lado, "o motivo-porque explica o projeto em função das vivências passadas do ator, [...] o contexto de significados do motivo-porque é sempre uma explicação posterior ao acontecimento"25:26. "É uma categoria objetiva acessível ao observador que tem de reconstruir a partir do ato realizado"23:126.

Portanto, ao cuidar de uma criança após uma cirurgia cardíaca, o acompanhante tem algo em vista, ou seja, os motivospara desta ação. Foi através dessa ação que conseguimos apreender o típico da ação do acompanhante que traz a criança ao atendimento no ambulatório de cirurgia cardíaca.

0 típico da ação consiste em evidenciar o que há de original, específico e típico na vivência do acompanhante da criança que veio ao ambulatório de cirurgia cardíaca. Pode-se obter um tipo ideal ao acentuar unilateralmente um ou diversos pontos de vista e encadear uma multiplicidade de fenômenos dados isoladamente, difusos e discretos, que se encontra ora em grande ora em pequeno número, e que se ordena para formar um quadro homogêneo de pensamento ${ }^{22}$.

Desta forma, "as tipificações ao nível do senso comum [...] emergem, na experiência cotidiana do mundo, como pressupostos, sem qualquer formulação de julgamentos ou proposições claras, com sujeitos e predicados lógicos"23:118.

Nesse sentido, os acompanhantes "lançam mão de seus conhecimentos disponíveis, [...] enquanto sedimentação de suas experiências vivenciadas ou recebidas dos contemporâneos, para agir de forma semelhante a algo experienciado como uma tipificação já existente"25:26.

0 estudo foi realizado no ambulatório de um Instituto de Cardiologia estadual localizado na Cidade do Rio de Janeiro, mediante aprovação do mesmo e no Comitê de Ética em Pesquisa. Essa instituição foi escolhida por ser o meu campo de trabalho, local onde suscitaram minhas inquietações relacionadas ao assunto em questão, o que para a fenomenologia é de suma importância.

0 instrumento utilizado na captação das falas dos sujeitos do estudo foi a entrevista fenomenológica.

Carvalho ${ }^{27: 37}$ esclarece que este tipo de entrevista "busca uma linguagem que seja 'fala originária', 'fala' esta que possibilite a mediação com o outro e a comunicação com 0 mundo". Na abordagem fenomenológica, valorizam-se todos os gestos, tonalidades de voz e momentos de silêncio inseridos no discurso do entrevistado, onde tudo é descrito para melhor compreensão dos significados. "0 entendimento de seu pensamento é a compreensão de um certo estilo de existir [...]". Portanto, para compreender o pensamento do entrevistado, é "preciso realizar uma comunhão com quem fala".

Como critério de inclusão, utilizaram-se os acompanhantes de crianças que já realizaram o procedimento cirúrgico e que retornaram para a consulta ambulatorial, não importando se foi a primeira vez ou não.

As falas foram captadas através da entrevista fenomenológica e gravadas mediante a autorização, utilizandose a seguinte questão de aproximação aos sujeitos do estudo: 
Qual o seu parentesco com a criança? e a questão orientadora do estudo que visa à captação do motivo-para, 0

\section{que espera da consulta no ambulatório?}

0 número de entrevistadas não foi determinado a priori. As entrevistas foram realizadas até o momento em que as falas se tornaram repetitivas; ou seja, quando houve a saturação nos achados, a similaridade entre as falas, e foi possível evidenciar a caracterização do motivo-para destas acompanhantes procurarem o ambulatório, a coleta de depoimentos se encerrou.

\section{O TÍPICO DA AÇÃO DOS ACOMPANHANTES DE CRIANÇA SUBMETIDA À CIRURGIA CARDÍACA}

Tendo em vista a utilização da abordagem fenomenológica como método para apoiar a análise compreensiva do estudo, fizemos uma leitura atentiva das falas das acompanhantes de crianças que retornaram para a consulta após a cirurgia cardíaca. Desta forma, surgiu a categoria que nos remetem ao motivo-para dessas pessoas quando retornam para a consulta independente do tempo decorrido da cirurgia. Assim, o típico da ação da acompanhante de criança que se submeteu à cirurgia cardíaca é "obter uma boa notícia", como podemos observar nos relatos a seguir.

Espero sair daqui sempre com uma boa notícia. (Sra. Incrível)

Espero ouvir que ela não precisa mais voltar, apesar de estar tão bem... é isso que eu quero ouvir, a melhora. (Rapunzel)

\section{Referências}

1. Ministério da Saúde (BR). Visita aberta e direito a acompanhante. Brasília (DF); 2004. 19p. [citado 22 jul 2007]. Disponível em: http:/ /dtr2001.saude.gov.br/editora/produtos/impressos/folheto/ 04_1160_FL.pdf.

2. Sales TCR, Maria VLR. Sentimento das mães de crianças submetidas à primeira cirurgia cardíaca no período trans - operatório. Arq Bras Cardiol [periódico on-line] 1999; [citado 21 set 2006].

Disponível em: http://www.arquivosonline.com.br/pesquisartigos/ Pdfs/TLS/1999/T468.pdf.

3. Gomes GC, Lunardi F. A família na unidade pediátrica: uma unidade que se cuida, uma unidade a ser cuidada. Texto\& Contexto Enferm 2000 maio/jun; 9 (2): 28 - 38.

4. Ministério da Saúde (BR). Estatuto da criança e do adolescente. Brasília (DF); 1991. 110 p.

5.Collet N, Rocha SMM. Criança hospitalizada: mãe e enfermagem compartilhando o cuidado. Rev Latino-am Enfermagem 2004 mar/ abr; 12 (2): 191-97.

6. Pettengill MAM, Angelo M. Family vulnerability: concept development. Rev Latino-am Enfermagem. [on-line] 2005; [citado 22 out2006]; 13 (6). Disponível em: http://www.scielo.br/scielo.php?script=sci_arttext\&pid=S010411692005000600010 \&lng=en\&nrm=iso.

7. Bowlby J. Apego e perda. Tradução de A. Cabral. São Paulo (SP): Martins Fontes; 1990.
Ouvir coisa boa... que ela fale que meu filho esteja bem... bem de saúde. (Bela Adormecida)

\section{CONSIDERAÇÕES FINAIS}

Ao refletir sobre o estudo, percebemos que a existência de um acompanhamento da enfermagem aos acompanhantes de crianças submetidas à cirurgia cardíaca faz-se necessário, desde que essas pessoas chegam na unidade hospitalar.

Nesse momento, é importante destacar que algumas orientações pertinentes à internação, à doença e à cirurgia são fundamentais. Durante o período de internação, realizar "grupos de apoio", onde o enfermeiro e os acompanhantes possam ter um momento para esclarecer dúvidas e minimizar ansiedades, é uma atitude que consideramos que deva fazer parte do cotidiano profissional. Esses procedimentos são redutores de ansiedade, pois os acompanhantes passam a ter esclarecimentos sobre a instituição e a situação da criança. Vale lembrar que a participação da equipe de enfermagem do centro cirúrgico e do CTI pós-operatório, falando sobre os setores, conversando sobre como funciona, proporcionando uma visitação dos acompanhantes, sem dúvida, pode ser uma excelente estratégia de acolhimento.

Este estudo nos fez pensar sobre a ação do enfermeiro ao implementar a consulta ambulatorial das crianças após a cirurgia cardíaca como uma estratégia importante na ação da Enfermagem Pediátrica visando à inclusão do acompanhante como cliente desse seu fazer cotidiano.

8. Castro EK, Piccinini CA. Implicações da doença orgânica crônica na infância para as relações familiares: algumas questões teóricas. Psicol reflex crit [on-line] 2002; [citado 23 set 2005]; 15 (3): 625-35. Disponível em: http://www.psicologia.ufrgs.br/gideb/piccinini/artp.htm\#doe.

9. Souza D0, Cosmo M, Pimentel RC. 0 grupo operativo: terapêutica eficaz para mães de crianças com cardiopatias congênitas. Rev SOCERJ. [on-line] 2003 jul; [citado 15 set 2005]; 16: 140. Disponível em: http://www.socerj.org.br/temas/19psico.pdf.

10. Lopes LM et al. Eco fetal: indicações. Rev Soc Cardiol Estado São Paulo 1999 set/out; 9 (5): 720-23.

11. LenfantC. Report of the task force on research in pediatric cardiovascular disease. Circulat [on-line] 2002 ago; [citado 15 set 2005] 106:1037. Disponível em: http://circ.ahajournals.org/cgi/content/full/106/9/1037.

12. Fortuna P. Pós-operatório imediato em cirurgia cardíaca. São Paulo (SP): Atheneu; 2000.

13. Ministério da Saúde (BR). Estatuto da criança e do adolescente. Brasília (DF); 1991.

14. Oliveira I, Angelo M. Vivenciando com o filho a passagem difícil e reveladora: a experiência da mãe-acompanhante. Rev Esc Enferm USP 2000 jun; 34 (2): 202-18.

15. Queiróz MVO, Barroso MGT. Qualidade de vida da mãe/ acompanhante de criança hospitalizada. Texto \& Contexto Enferm 1999 set/dez; 8 (3): 147-61.

16. Collière MF. Cuidar... a primeira arte da vida. Lisboa (P0): Lusociências; 2003. 
17. Silveira A0, Angelo MA experiência de interação da família que vivencia a doença e hospitalização da criança. Rev Latino-am Enfermagem. [on-line] 2006; [citado 25 set 2007]; 14 (6). Disponível em: http://www.scielo.br/scielo.php?script=sci_arttext\&pid=S0104$11692006000600010 \&$ lng =pt\&nrm=iso.

18. Coltro A. A fenomenologia: um enfoque metodológico para além da modernidade. Cad Pesqui Adm 2000; 1(11): 37- 45.

19. Polit DF, Hungler BP. Fundamentos de pesquisa em enfermagem. $3^{\mathrm{a}}$ ed. Porto Alegre (RS): Artes Médicas; 1995.

20. Bello AA. Fenomenologia e ciências humanas: psicologia, história e religião. Tradução de Miguel Mahfoud e Marina Massimi. Bauru (SP): EDUSC; 2004.

21. Josgrilberg RS. A fenomenologia como novo paradigma de uma ciência do existir. In: Pokladek DD, organizador. A fenomenologia do cuidar. São Paulo (SP): Vetor Ed Psico-pedagógica; 2004.

22. Capalbo C. Metodologia das ciências sociais: a fenomenologia de Alfred Schutz. Londrina (PR): UEL; 1998.
23. Wagner HR. Fenomenologia e relações sociais. Rio de Janeiro (RJ): Zahar; 1979.

24. Popim RC, Boemer MR. 0 cuidar em oncologia: diretrizes e perspectivas. São Paulo (SP): UNESP; 2006.

25. Rodrigues BMRD. 0 cuidar de crianças em creche comunitária: redimensionando o treinamento numa perspectiva compreensiva [tese de doutorado]. Rio de Janeiro (RJ): Escola de Enfermagem Anna Nery/UFRJ; 1996.

26. Panizza L. 0 pensamento de Alfred Schutz: sociologia fenomenológica. Rev Bras Filos 1981 abr/jun; 31 (122): 129.

27. Carvalho AS. Metodologia da entrevista: uma abordagem fenomenológica. $2^{\text {a }}$ ed. Rio de Janeiro (RJ): Agir; 1991.

\section{Notas}

aEste estudo faz parte da Dissertação de Mestrado realizado na Faculdade de Enfermagem da UERJ no período de 2006 a 2007. 